\title{
Oleifolioside B-mediated autophagy promotes apoptosis in A549 human non-small cell lung cancer cells
}

\author{
CHENG-YUN JIN ${ }^{1 *}$, HAI YANG YU ${ }^{2 *}$, CHEOL PARK $^{3}$, MIN HO HAN $^{4,10}$, SU HYUN HONG ${ }^{4}$, \\ KYOUNG-SOOK KIM ${ }^{2}$, YOUNG-CHOON LEE ${ }^{2}$, YOUNG-CHAE CHANG ${ }^{5}$, JAEHUN CHEONG $^{6}$, \\ SUNG-KWON MOON ${ }^{7}$, GI-YOUNG KIM ${ }^{8}$, HYUNG-IN MOON ${ }^{2}$, WUN-JAE KIM ${ }^{9}$, \\ JAI-HEON LEE ${ }^{2}$ and YUNG HYUN CHOI ${ }^{4,10}$
}

\footnotetext{
${ }^{1}$ School of Pharmaceutical Science, Zhengzhou University, Henan 450001, P.R. China; ${ }^{2}$ College of Natural Resources

and Life Science, BK21 Center for Silver-Bio Industrialization, Dong-A University, Busan 604-714;

${ }^{3}$ Department of Molecular Biology, College of Natural Sciences, Dongeui University, Busan 614-714;

${ }^{4}$ Department of Biochemistry, Dongeui University College of Oriental Medicine, Busan 614-052; ${ }^{5}$ Research Institute of Biomedical Engineering and Department of Medicine, Catholic University of Daegu School of Medicine, Daegu 705-718;

${ }^{6}$ Department of Molecular Biology, College of Natural Sciences, Busan National University, Busan 609-735;

${ }^{7}$ School of Food Science and Technology, Chung-Ang University, Ansung 456-756; ${ }^{8}$ Laboratory of Immunobiology,

Department of Marine Life Sciences, Jeju National University, Jeju 690-756; ${ }^{9}$ Department of Urology,

Chungbuk National University College of Medicine, Cheongju 361-763; ${ }^{10}$ Anti-Aging Research Center and Blue-Bio Industry Regional Innovation Center, Dongeui University, Busan 614-714, Republic of Korea
}

Received August 7, 2013; Accepted September 30, 2013

DOI: 10.3892/ijo.2013.2143

\begin{abstract}
The biochemical mechanisms of cell death by oleifolioside B (OB), a cycloartane-type triterpene glycoside isolated from Dendropanax morbifera Leveille, were investigated in A549 human lung carcinoma cells. Our data indicated that exposure to OB led to caspase activation and typical features of apoptosis; however, apoptotic cell death was not prevented by z-VAD-fmk, a pan-caspase inhibitor, demonstrating that OB-induced apoptosis was independent of caspase activation. Subsequently, we found that OB increased autophagy, as indicated by an increase in monodansylcadaverine fluorescent dye-labeled autophagosome formation and in the levels of the autophagic form of microtubule-associated protein 1 light chain 3 and Atg3, an autophagy-specific gene, which is associated with inhibiting phospho-nuclear factor
\end{abstract}

Correspondence to: Dr Jai-Heon Lee, College of Natural Resources and Life Science, BK21 Center for Silver-Bio Industrialization, Dong-A University, Busan 604-714, Republic of Korea

E-mail: jhnlee@dau.ac.kr

Dr Yung Hyun Choi, Department of Biochemistry, Dongeui University College of Oriental Medicine, Busan 614-052, Republic of Korea

E-mail: choiyh@deu.ac.kr

*Contributed equally

Key words: oleifolioside B, apoptosis, autophagy, nuclear factor erythroid 2-related factor 2 erythroid 2-related factor 2 (Nrf2) expression. However, pretreatment with bafilomycin $\mathrm{A} 1$, an autophagy inhibitor, attenuated OB-induced apoptosis and dephosphorylation of Nrf2. The data suggest that OB-induced autophagy functions as a death mechanism in A549 cells and OB has potential as a novel anticancer agent capable of targeting apoptotic and autophagic cell death and the Nrf2 signaling pathway.

\section{Introduction}

Programmed cell death (PCD) plays an essential role in regulating various biological processes, including morphogenesis, maintaining tissue homeostasis, and eliminating damaged and infected cells. Two major forms of PCD have been classified as apoptosis and autophagy (1-3). Apoptotic cell death (type I PCD) is characterized by membrane blebbing, chromosomal DNA fragmentation, cell shrinkage and the formation of apoptotic bodies (4-6). Autophagic cell death (type II PCD) is a catabolic process in which cytosolic macromolecules and damaged organelles are sequestered in double-membrane autophagosomes, which subsequently fuse with lysosomes for degradation by forming acidic autophagolysosomes $(7,8)$. Different from apoptosis, autophagy may contribute to cell survival or death. Although apoptosis and autophagy are two distinct biological processes, crosstalk exists between them (9-11).

Lung cancer is the most common cause of cancer-related morbidity and mortality in men and women around the world, accounting for approximately $30 \%$ of all cancer deaths (12). Lung cancers are generally classified into two histological types, small cell lung cancer and non-small cell lung cancer 
(NSCLC). NSCLC accounts for approximately $85 \%$ of all lung cancers. Most lung cancers are closely related to an advanced stage at diagnosis with a poor prognosis. Over the last few decades, chemotherapy has improved the outcome for patients with late stage NSCLC, but only slightly $(13,14)$. Therefore, novel and more effective antitumor agents must be explored and developed.

Dendropanax morbifera Leveille has been used in traditional medicine to treat several diseases, such as headache, infectious diseases and skin diseases. Previous studies have shown that the components of this plant have many pharmacological activities, including anti-complement, anti-diabetic and anti-atherogenic properties (15-17). Recently, oleifolioside B (OB), a cycloartane-type glycoside, was isolated from the lower stem parts of D. morbifera, which has anti-plasmodial activity in vitro (18). However, little is known regarding the anticancer activity of $\mathrm{OB}$ or its signal molecular mechanisms in cancer cells. In this present study, we investigated for the first time the anticancer mechanisms by which OB induces apoptosis and autophagy in A549 NSCLC cells.

\section{Materials and methods}

Materials. 3-(4,5-dimethylthiazol-2-yl)-2,5-diphenyltetrazolium bromide (MTT), bafilomycin A1, 4,6-diamidino-2-phenylindole (DAPI), monodansylcadaverine (MDC) and doxorubicin were purchased from the Sigma-Aldrich Chemical Co. (St. Louis, MO). Antibodies specific for actin, Bad, Bax, Bcl-2, Bcl-xL, Bid, caspase-3, caspase-8, caspase-9, cIAP-1, cIAP-2, death receptor (DR) 4, DR5, Fas, Fas legend (FasL), Fas-associated death domain (FADD), cellular FLICE-like inhibitory protein (c-FLIP), nuclear factor erythroid 2-related factor 2 (Nrf2), poly(ADP-ribose) polymerase (PARP), survivin, XIAP, heme oxygenase 1 (HO-1) and goat anti-rabbit IgG-FITC were obtained from Santa Cruz Biotechnology (Santa Cruz, CA). Antibodies specific for Atg3, Atg5, Atg7, Atg12, Beclin-1 and microtubule-associated protein 1 light chain 3 (LC3) were purchased from Cell Signaling (Beverly, MA). The antibody specific for phospho (p)-Nrf2 was purchased from Epitomics (Burlingame, CA). Peroxidase-labeled donkey anti-rabbit and sheep anti-mouse immunoglobulins and an enhanced chemiluminescence (ECL) kit were purchased from Amersham (Arlington Heights, IL). Caspase activity assay kits were purchased from R\&D Systems (Minneapolis, MN). z-VAD-fmk was purchased from Calbiochem (San Diego, CA). OB was kindly provided by Professor Hyung-In Moon of Dong-A University (Busan, Republic of Korea) (18) and dissolved in dimethyl sulfoxide (DMSO) as a stock solution at $30 \mathrm{mM}$ concentration. Dilutions were made in Dulbecco's modified Eagle's medium (DMEM, Gibco-BRL, Gaithersburg, MD).

Cell culture and cell viability assay. The A549 cell line was obtained from the American Type Culture Collection (Rockville, MD) and cultured in DMEM supplemented with $10 \%$ heat-inactivated fetal bovine serum (FBS), $2 \mathrm{mM}$ glutamine, $100 \mathrm{U} / \mathrm{ml}$ penicillin, and $100 \mu \mathrm{g} / \mathrm{ml}$ streptomycin (Gibco-BRL). Cell viability was measured based on formation of blue formazan metabolized from colorless MTT by mitochondrial dehydrogenases, which are active only in live cells.
Flow cytometric analysis. To analyze the percentage of apoptotic cells, cells were collected, washed with cold phosphate-buffered saline (PBS), and fixed in $75 \%$ ethanol at $4^{\circ} \mathrm{C}$ for $30 \mathrm{~min}$. The DNA content of the cells was measured using a DNA staining kit (CycleTEST PLUS Kit, Becton-Dickinson, San Jose, CA). Propidium iodide (PI)-stained nuclear fractions were obtained by following the kit protocol. The cells were then filtered through $35-\mathrm{mm}$ mesh, and DNA content fluorescence was determined using a FACSCalibur flow cytometer within $1 \mathrm{~h}$. The cellular DNA content was analyzed with CellQuest software (Becton-Dickinson).

DNA fragmentation assay. After OB treatment, the cells were lysed in a buffer containing $10 \mathrm{mM}$ Tris- $\mathrm{HCl}, \mathrm{pH} 7.4$, $150 \mathrm{mM} \mathrm{NaCl}, 5 \mathrm{mM}$ EDTA and $0.5 \%$ Triton X-100 for $1 \mathrm{~h}$ at room temperature. The lysates were vortexed and cleared by centrifugation at $15,000 \mathrm{rpm}$ for $10 \mathrm{~min}$ at $4^{\circ} \mathrm{C}$. The DNA in the supernatant was extracted using a 25:24:1 (v/v/v) equal volume of neutral phenol:chloroform:isoamyl alcohol. To assay the DNA fragmentation pattern, samples were loaded onto $1.0 \%$ agarose gel containing $0.1 \mu \mathrm{g} / \mathrm{ml}$ ethidium bromide $(\mathrm{EtBr})$ and electrophoresis was carried out.

Caspase activity assay. The enzymatic activity of the caspases was assayed using a colorimetric assay kit according to the manufacturer's protocol. The cells were incubated in the absence and presence of OB for the indicated times. The cells were harvested and lysed in a lysis buffer for $30 \mathrm{~min}$ on an ice bath. Lysed cells were centrifuged at 14,000 rpm for $20 \mathrm{~min}$, and equal amounts of protein $(100 \mu \mathrm{g}$ per $50 \mu \mathrm{l})$ were incubated with $50 \mu 1$ reaction buffer and $5 \mu 1$ colorimetric tetrapeptides, Asp-Glu-Val-Asp (DEVD)-p-nitroaniline (pNA) for caspase-3, Ile-Glu-Thr-Asp (IETD)-pNA for caspase-8, and Leu-Glu-His-Asp (LEHD)-pNA for caspase-9, at $37^{\circ} \mathrm{C}$ for $2 \mathrm{~h}$ in the dark. Caspase activity was determined by measuring the changes in absorbance at $405 \mathrm{~nm}$ using an ELISA reader.

MDC staining. To observe autophagy formation, A549 cells were grown on glass coverslips for $24 \mathrm{~h}$. The cells were incubated in the absence and presence of $\mathrm{OB}$ for the indicated times, and then the cells were treated with $0.05 \mathrm{mM} \mathrm{MDC}$ at $37^{\circ} \mathrm{C}$ in $5 \% \mathrm{CO}_{2}$ for $1 \mathrm{~h}$. The cells were then fixed with $4 \%$ paraformaldehyde in PBS for $10 \mathrm{~min}$. The cellular changes were analyzed with a fluorescence microscope.

Protein extraction and western blot analysis. Whole-cell protein extracts from A549 cells were prepared with cell lysis buffer (20 mM sucrose, $1 \mathrm{mM}$ EDTA, $20 \mu \mathrm{M}$ Tris-HCl, pH 7.2, $1 \mathrm{mM}$ DTT, $10 \mathrm{mM} \mathrm{KCl}, 1.5 \mathrm{mM} \mathrm{MgCl}_{2}$ and $5 \mu \mathrm{g} / \mathrm{ml}$ aprotinin) for $30 \mathrm{~min}$. Cells were disrupted by sonication and extracted at $4^{\circ} \mathrm{C}$ for $30 \mathrm{~min}$. The protein extracts were quantified using the Bio-Rad kit (Pierce Biotechnology, Rockford, IL). For western blot analysis, lysate proteins (30-50 $\mu \mathrm{g}$ ) were resolved over sodium dodecyl sulfate (SDS)-polyacrylamide gel electrophoresis and transferred onto nitrocellulose transfer membranes (Schleicher \& Schuell, Keene, NH). Specific proteins were detected with an ECL Western blotting kit according to the recommended procedure. In a parallel experiment, cells were washed with ice-cold PBS and collected. Then cytoplasmic 

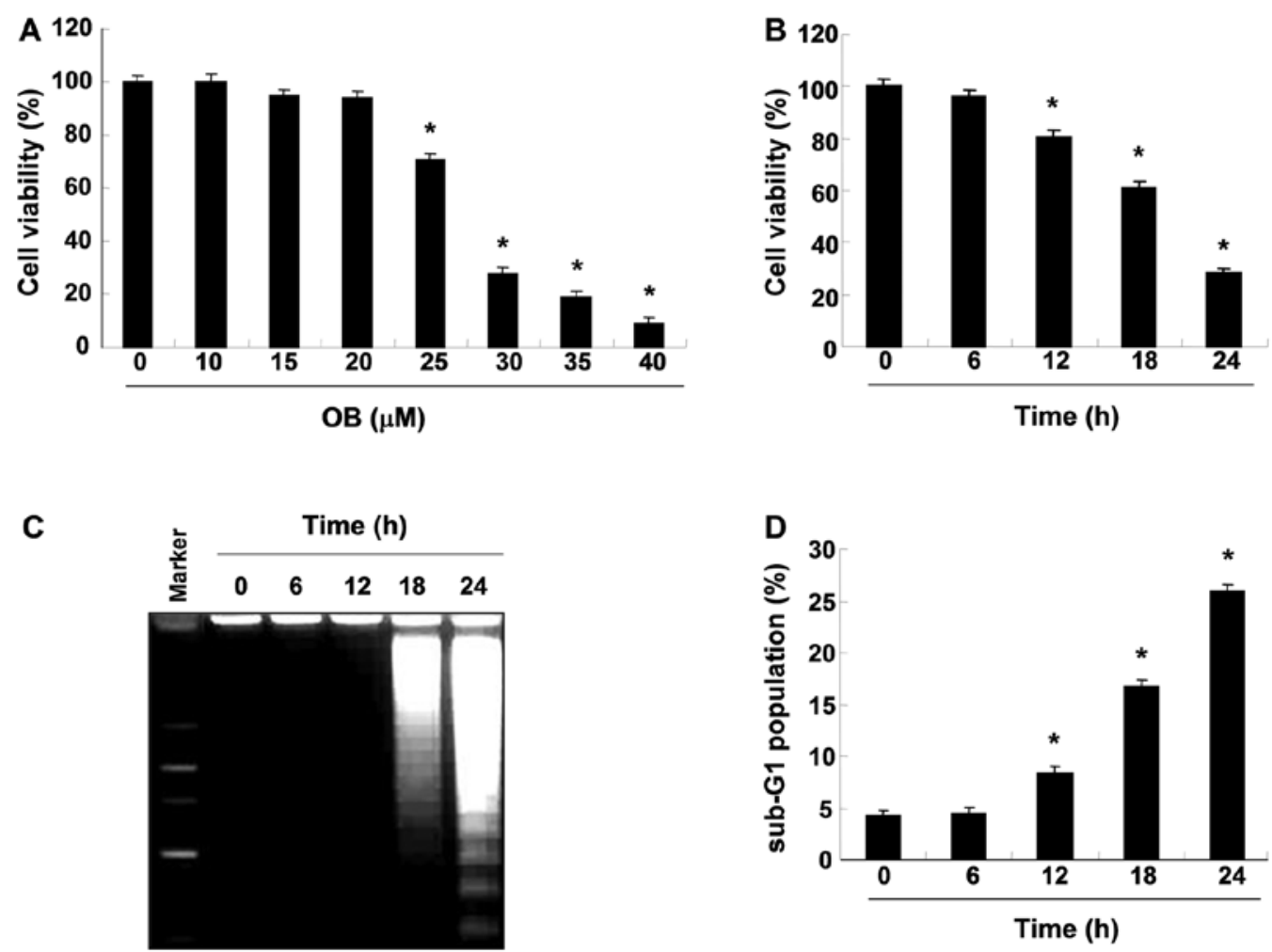

Figure 1. OB induces cell growth inhibition and apoptosis in A549 cells. (A and B) Cells were seeded at $2 \times 10^{5} \mathrm{cells} / \mathrm{ml}$ for $24 \mathrm{~h}$, and then treated with the indicated concentrations of $\mathrm{OB}$ for (A) $24 \mathrm{~h}$ or $40 \mu \mathrm{M} \mathrm{OB}$ for the indicated durations (B). Then cell viability was determined with MTT assay. (C) After the cells were treated with $30 \mu \mathrm{M} \mathrm{OB}$ for the indicated durations, the cells were stained with DAPI solution for $10 \mathrm{~min}$ and then photographed with a fluorescence microscope using a blue filter (x400). (C) For the DNA fragmentation analysis, genomic DNA was extracted from cells grown under the same conditions as (B), separated by $1.0 \%$ agarose gel electrophoresis, and visualized under UV light after staining with EtBr. The marker indicates a size marker the DNA ladder. (D) To quantify the degree of apoptosis induced by OB, cells were evaluated with a flow cytometer for sub-G1 DNA content, which represents the cells undergoing apoptotic DNA degradation. Each point represents the mean \pm SD of three independent experiments. "Significantly different from control $(\mathrm{p}<0.05)$.

and nuclear proteins were prepared using NE-PER Nuclear and Cytoplasmic Extraction Reagents (Pierce Biotechnology).

Immunocytochemistry. Cells were grown on coverslips and treated as indicated. Cells were washed twice in PBS, fixed with 4\% paraformaldehyde in PBS at room temperature for $30 \mathrm{~min}$, and then permabilized with $0.25 \%$ Triton X-100 solution for $10 \mathrm{~min}$. The cells were subsequently incubated in a blocking solution of $1 \%$ bovine serum albumin (BSA) and incubated with primary antibodies at room temperature for $1 \mathrm{~h}$. After the incubation period, the samples were rinsed four times with PBS and then incubated with the secondary anti-rabbit-FITC diluted 1:200 in buffer for $1 \mathrm{~h}$ at room temperature. Nuclei were stained with $1 \mu \mathrm{g} / \mathrm{ml}$ DAPI, and then captured using a Zeiss LSM 510 laser scanning confocal device (Carl Zeiss).

Statistical analysis. All data are expressed as mean \pm SD. The significant differences between the groups were determined using an unpaired Student's t-test. A value of $\mathrm{p}<0.05$ was considered significant. All figures shown represent results from at least two independent experiments with a similar pattern.

\section{Results}

Induction of apoptosis by $O B$ in $A 549$ cells. To investigate the effects of OB on cell viability in A549 cells, cells were treated with OB and subjected to an MTT assay. As shown in Fig. 1A and B, treatment with OB significantly reduced cell viability in a concentration- and time-dependent manner. Subsequently, to examine whether OB inhibits the proliferation of A549 cells by inducing apoptosis, genomic DNA was extracted from cells and agarose gel electrophoresis were assessed. As indicated Fig. 1C, treatment with OB time-dependently induced DNA fragmentation, a hallmark of apoptosis, in a time-dependent manner. In addition, flow cytometric analysis also revealed that treatment with $\mathrm{OB}$ increased the accumulation of cells at the apoptotic sub-G1 phase in a time-dependent manner (Fig. 1D). Taken together, these results indicate that the cytotoxic effects observed in response to $\mathrm{OB}$ are associated with the induction of apoptotic cell death in A549 cells.

Modulation of apoptosis regulatory proteins by $O B$ in $A 549$ cells. To determine which pathway was involved in the apoptosis induction of OB-treated A549 cells, the expression levels of death receptor-related proteins and the Bcl-2 and IAP family of proteins were determined with western blot analysis to measure the expression of the proteins. As shown in Fig. 2, exposure to $\mathrm{OB}$ led to a significant reduction in the anti-apoptotic protein cIAP-2, survivin and c-FLIP in a time-dependent fashion. However, OB treatment resulted in a time-dependent increase in the level of the pro-apoptotic FasL proteins. Under these conditions, although we did not 

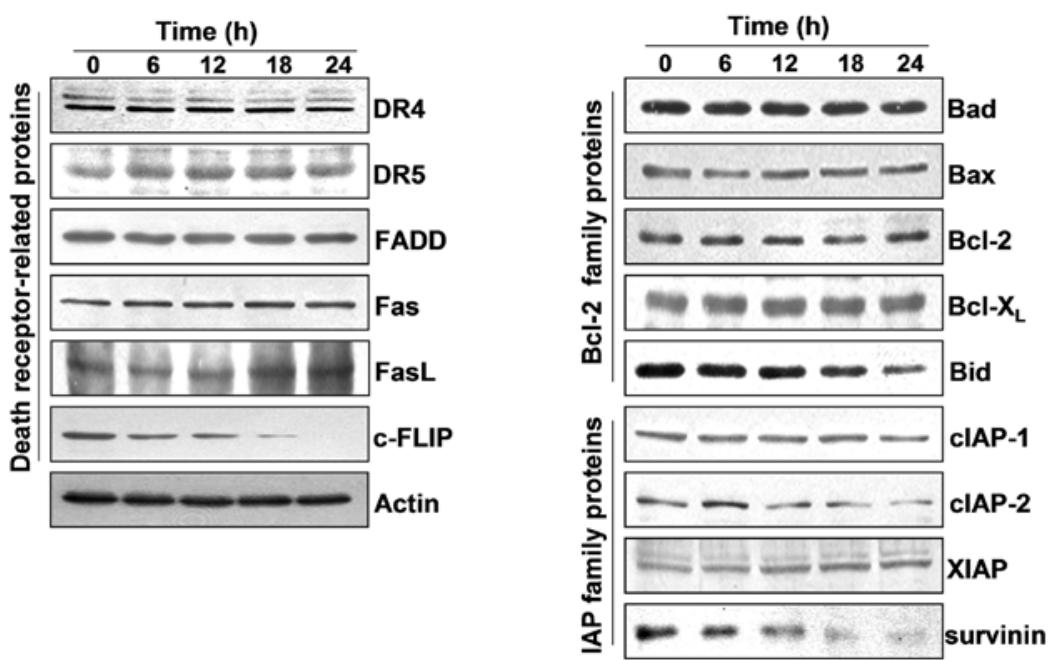

Figure 2. Modulation of apoptosis-related protein expression by OB in A549 cells. A549 cells were treated with $30 \mu \mathrm{M}$ OB for the indicated durations. The cells were lysed, and then the cellular proteins were separated with SDS-polyacrylamide gels and transferred onto nitrocellulose membranes. The membranes were probed with the indicated antibodies. Proteins were visualized using an ECL detection system. Actin was used as an internal control.

detect the truncated form of the pro-apoptotic protein Bid, a BH3-only pro-apoptotic member of the Bcl-2 family, our results indicate that $\mathrm{OB}$ treatment caused time-dependent downregulation of the whole form of the Bid proteins, which reflects Bid cleavage and activation.

Caspase activation by $O B$ in $A 549$ cells. To investigate whether OB-induced apoptosis in A549 cells involves the caspase cascade pathway, the caspase expression levels and activity were determined. As shown in Fig. 3A, western blot analyses indicated that the active forms of caspase- 3 and -8 increased and the expression of pro-caspase- 9 decreased in a time-dependent manner following OB treatment. For further quantification of the proteolytic activation of caspases, protein in the lysates of cells treated with OB was normalized and then assayed for in vitro activities using fluorogenic substrates. As indicated in Fig. 3B, treatment with $\mathrm{OB}$ resulted in a time-dependent increase in caspase activity $(-3,-8$ and -9$)$ compared with the control cells, which was associated with the progressive proteolytic cleavage products of PARP, an activated caspase- 3 substrate protein. To further investigate the significance of caspase activation in OB-induced apoptosis, A549 cells were pretreated with $\mathrm{z}$-VAD-fmk, a broad-spectrum caspase inhibitor, for $1 \mathrm{~h}$, followed by treatment with $30 \mu \mathrm{M}$ OB for $24 \mathrm{~h}$. Interestingly, pretreatment with z-VAD-fmk did not restore cell viability compared with control (Fig. 4A), and failed to suppress the OB-induced apoptosis (Fig. 4B). However, under the same condition, z-VAD-fmk significantly suppressed doxorubicininduced growth inhibition and apoptosis (Fig. 4). Taken together, these results suggest that $\mathrm{OB}$-induced apoptosis was independent of caspase activation in A549 cells.

Induction of autophagy by $O B$ in $A 549$ cells. As shown in Fig. 5A, based on our finding that extensive cytoplasm vacuolization compared with control cells appeared in cytosol after $\mathrm{OB}$ treatment, we hypothesized that the vacuoles might be associated with autophagy. For this study, we used MDC,
A
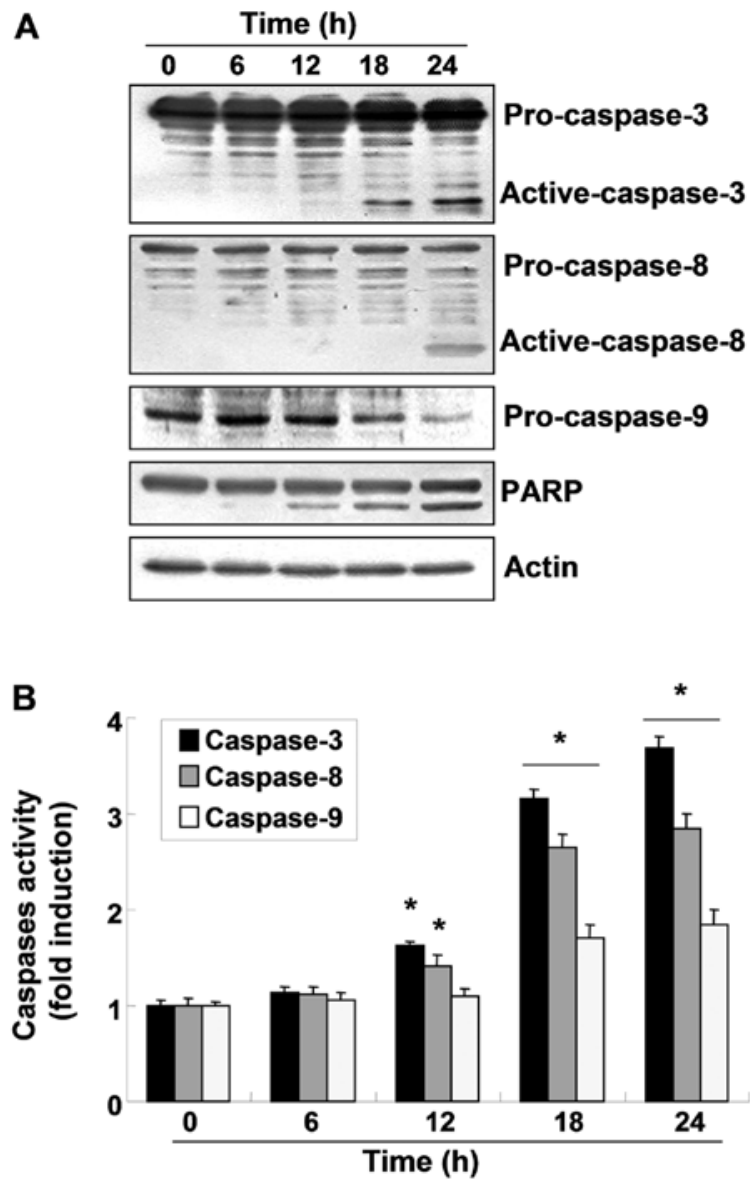

Figure 3. Activation of caspases and degradation of PARP by OB in A549 cells (A) Cells treated with $30 \mu \mathrm{M}$ OB for the indicated durations were lysed. Then the cellular proteins were separated with SDS-polyacrylamide gels and transferred onto nitrocellulose membranes. The membranes were probed with the indicated antibodies. Proteins were visualized using an ECL detection system. Actin was used as an internal control. (B) Cells grown under the same conditions as (A) were lysed, and aliquots (50 $\mu \mathrm{g}$ protein) were assayed for in vitro caspase-3, -8 and -9 activity using DEVD-pNA, IETD-pNA and LEHD-pNA as the substrate, respectively. The released fluorescent products were measured. Data are expressed as the mean \pm SD of three independent experiments. The significance was determined with Student's t-test (" $\mathrm{p}<0.05$ vs. untreated control). 

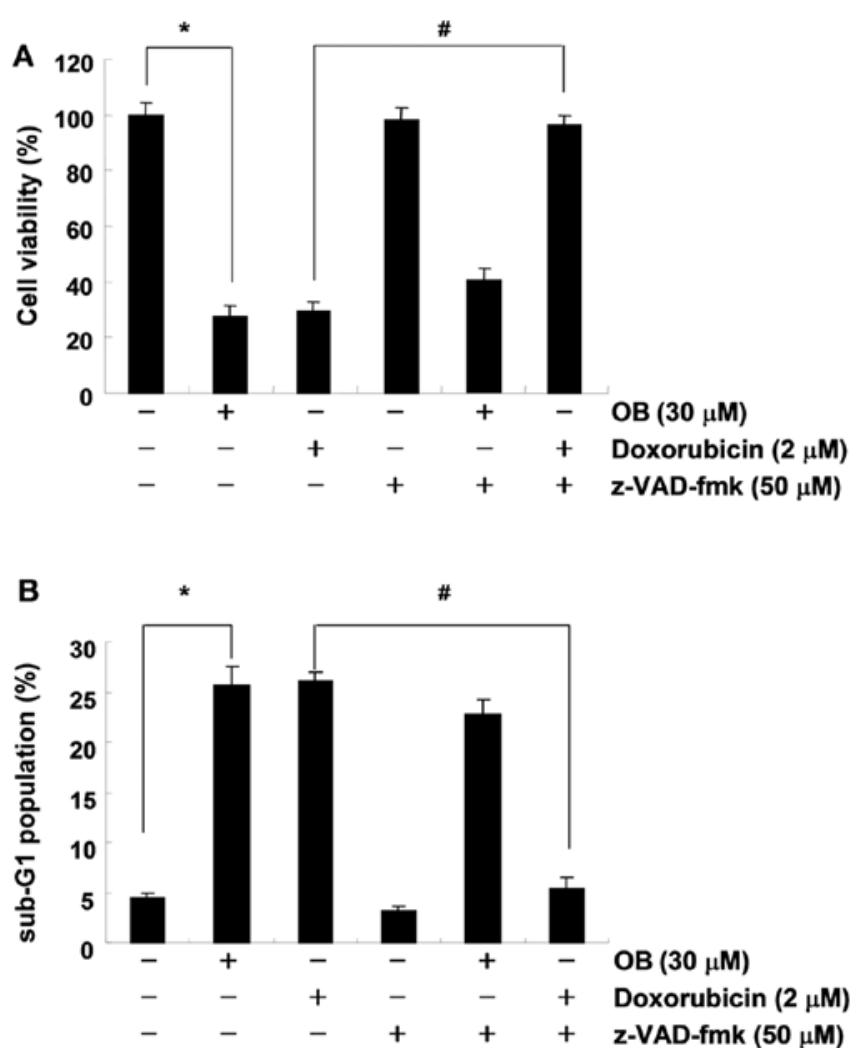

Figure 4. Effects of a pan-caspase inhibitor, z-VAD-fmk, on OB-induced apoptosis in A549 cells. (A) Cells were pretreated $50 \mu \mathrm{M}$ z-VAD-fmk for $1 \mathrm{~h}$, and then treated with $30 \mu \mathrm{M}$ OB or $2 \mu \mathrm{M}$ doxorubicin as the positive control for $24 \mathrm{~h}$. Cell viability was determined with MTT assay. (B) A549 cells grown under the same conditions as (A) were evaluated with a flow cytometer for sub-G1 DNA content. Each point represents the mean \pm SD of three independent experiments. "Significantly different from control $(\mathrm{p}<0.05)$. "Significantly different from doxorubicin-treated cells $(\mathrm{p}<0.05)$.

a commonly used autophagic dye, as a more specific marker for autophagy. The results indicated that untreated control cells presented diffused staining, but OB treatment clearly resulted in an extensive punctuate MDC staining pattern (Fig. 5B). However, LC3, another typical marker of autophagy, exists in the cytosol and is called LC3-I, after its C-terminal region, which is cleaved through post-translational modification. When autophagosomes are formed, LC3-I changes to LC3-II through conjugation with phosphatidylethanolamine and is recruited into the autophagosome membrane. That is why LC3-II accumulation is a critical marker of autophagy (19). As shown in Fig. 6C, OB treatment induced an increase in LC3-II expression. In addition, treatment with OB strongly increased the essential autophagosome-regulatory gene expression of $\operatorname{Atg} 3$, and slightly increased the gene expression of Atg5, but not Atg7 and Atg12. We also found an accumulation of LC3 puncta following OB treatment through fluorescence microscopic observation (Fig. 7A). Taken together, these results indicate that OB-induced cytoplasmic vacuoles are related to autophagy induction.

Modulation of Nrf2 in OB-treated A549 cells. Because many recent studies have reported that Nrf2 is involved in cancer cell survival and in the acquisition of drug resistance

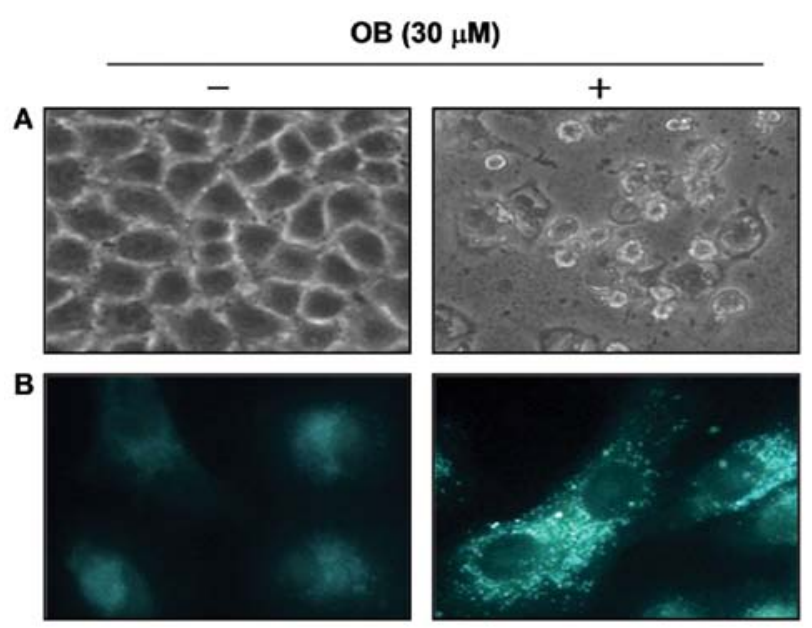

C

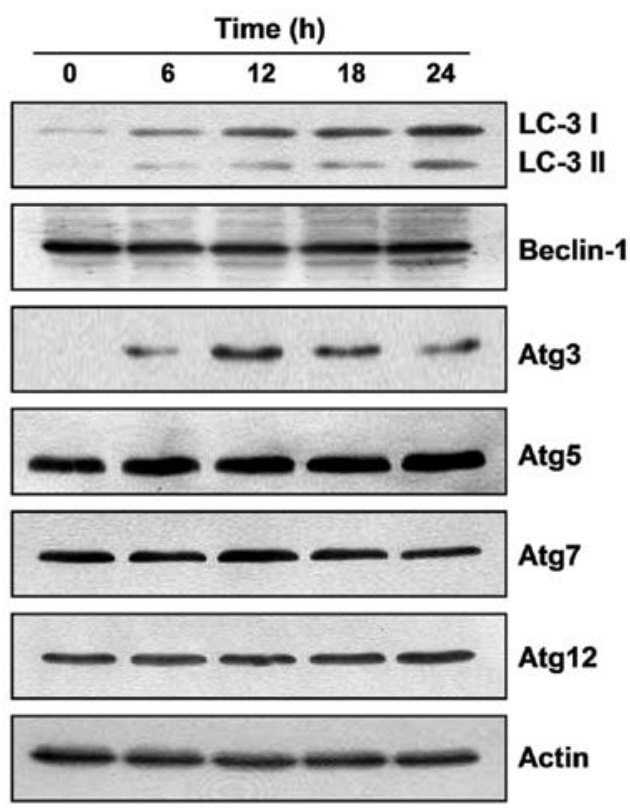

Figure 5. Induction of autophagy by OB in A549 cells. (A and B) Cells were incubated without or with $30 \mu \mathrm{M}$ OB for $24 \mathrm{~h}$. Then the cells were observed using an inverted microscope (A) or stained with MDC solution for $1 \mathrm{~h}$, and then photographed with a fluorescence microscope using a blue filter (B, x400). (C) The cells were treated with $30 \mu \mathrm{M}$ OB for the indicated durations, lysed, and then cellular proteins were separated with SDS-polyacrylamide gels and transferred onto nitrocellulose membranes. The membranes were probed with the indicated antibodies. Proteins were visualized using an ECL detection system. Actin was used as an internal control.

against anticancer therapies, we investigated whether the Nrf2 signaling pathway is involved in OB-caused cell death. As shown in Fig. 6, although exposure of cells to OB led to dephosphorylation of $\mathrm{Nrf} 2$ proteins without altering their total levels, Nrf2 and p-Nrf2, the active form of Nrf2, were translocated to the nucleus in response to OB treatment, which was associated with decreased expression of HO-1 whose transcription is regulated by Nrf2. Therefore, OB-induced cell death appeared to be responsible for nuclear translocation of p-Nrf2.

OB-induced autophagy as a death mechanism in A549 cells. To elucidate the molecular mechanism controlling the rela- 


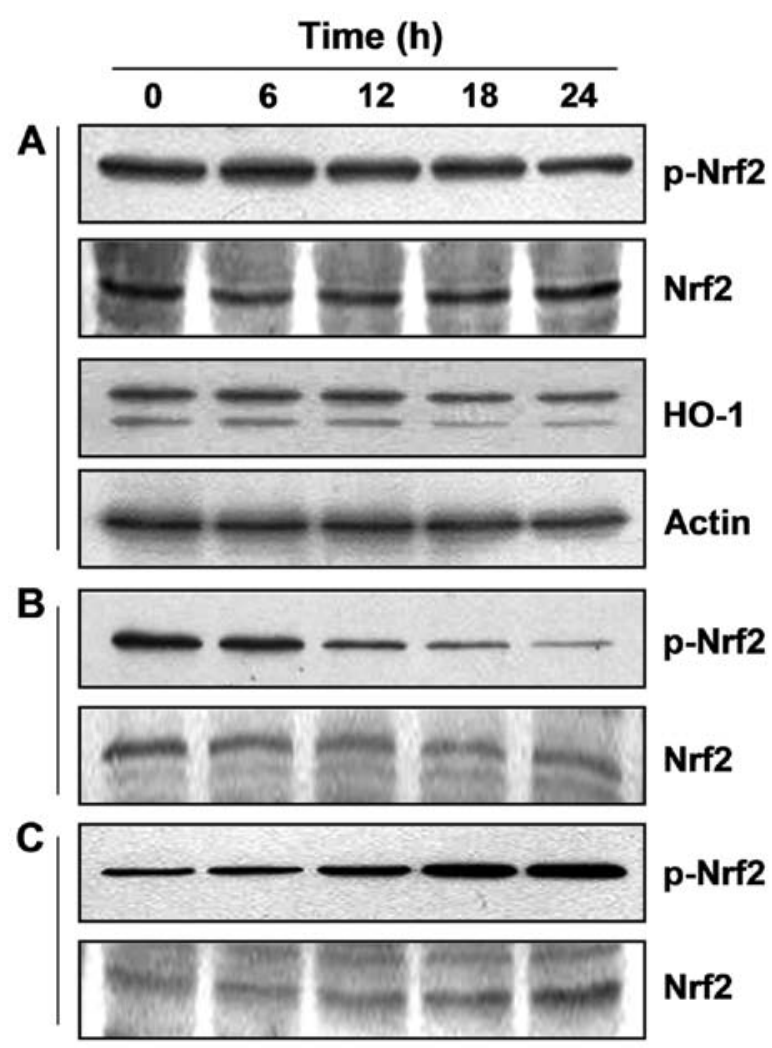

Figure 6. Effects of OB on the Nrf2 levels in A549 cells. Cells were treated with $30 \mu \mathrm{M}$ OB for the indicated time period. (A) Total cell lysate, (B) cytosolic, and $(C)$ nuclear proteins were extracted, and then equal amounts of the proteins were resolved on SDS-polyacrylamide gels and transferred to the nitrocellulose membranes. Proteins were detected with anti-p-Nrf2, Nrf2 and HO-1 antibodies and ECL detection. Actin was used as an internal control.

tionship between OB-induced apoptosis and autophagy, we next investigated whether OB-induced autophagy functioned as a survival mechanism or a death mechanism. As shown in Fig. 7A, pretreatment with bafilomycin A1, a well-known inhibitor of autophagosomal lysosome degradation, blocked the formation of OB-induced LC3 puncta, representing recruited LC3-II in the cytosol. Western blot analysis also showed that PARP cleavage, caspase-3 activation, and p-Nrf2 translocation were restored to levels seen in the control cells in response to bafilomycin A1 pretreatment, which was connected with significant blockage of OB-induced apoptosis and growth inhibition (Fig. 7C and D). Taken together, these results clearly show that $\mathrm{OB}$ treatment induces autophagy as a cell death mechanism in A549 cells.

\section{Discussion}

In the present study, we demonstrated that the growth inhibitory effects of $\mathrm{OB}$, a cycloartane-type triterpene glycoside isolated from $D$. morbifera, were associated with induction of apoptosis and autophagy in A549 NSCLC cells. Our data also suggested that $\mathrm{OB}$-induced autophagy functioned as a death mechanism, which was connected to the translocation of p-Nrf2 from cytoplasm to the nucleus.

Apoptotic cell death, type I PCD, is an important process that allows a cell to self-degrade to eliminate damaged cells $(4,5)$. There are two main apoptotic pathways: the intrinsic mitochondrial pathway and the extrinsic death receptor pathway. The intrinsic pathway, which is triggered by various extracellular and intracellular stresses, produces mitochondrial-mediated signals, causing the mitochondrial permeability transition pore and the release of pro-apoptotic proteins such as cytochrome $c$. The release of cytochrome $c$ is essential for caspase- 9 activation. Activated caspase- 9 in turn activates the downstream effector caspase-3 and -7, which rapidly cleave intracellular substrates (4-6). The extrinsic signaling pathway initiates apoptosis through transmembrane receptor-mediated interactions. The best characterized cytoplasmic death receptors are Fas, DR4 and DR5. After the respective ligands bind to the receptors, an adapter protein called Fas-associated death domain (FADD) or TRAIL-associated death domain (TRADD) is recruited to the death receptor, forming DISC to activate pro-caspase- 8 . The activation of caspase- 8 is antagonized by c-FLIP, an enzymatically-inactive relative of caspase- 8 that binds to DISC. Therefore, the knockdown of c-FLIP augments DISC recruitment, activation and processing of caspase-8, thus enhancing effector-caspase stimulation and apoptosis $(20,21)$. Several pro-apoptotic and anti-apoptotic proteins are involved in the upstream and downstream of this process, such as IAP and Bcl-2 member proteins, as well as the expression of death receptors $(22,23)$. In our study, among the Bcl-2 family proteins, OB treatment markedly downregulated the whole form of the Bid proteins in a timedependent manner reflecting Bid cleavage and activation. OB also significantly inhibited the expression of c-FLIP without altering the DR4, DR4, FADD, Fas and FasL levels. Caspases are integral components of the apoptotic pathway. Although many reports have shown that chemotherapeutic agents induced apoptosis through the caspase-dependent pathway in cancer cells, apoptotic cell death can occur in a caspase-independent manner (24-26). In our case, OB induced activation of initiator caspases (caspase- 8 and -9) and an effector caspase (caspase-3), but did not restore cell viability and failed to suppress the OB-induced apoptotic cell deaths by treatment with z-VAD-fmk, a broad-spectrum caspase inhibitor. The results indicate that treatment with OB-induced apoptosis was caspase-independent of the death receptor signaling and mitochondria pathways in A549 cells.

In addition to caspase-dependent cell death, some cell death processes occur in a caspase-independent manner. Autophagy (type II PCD) is a cellular process characterized by sequestration of part of the cell cytoplasm, including long-lived protein organelles, in autophagic vesicles and their delivery to and subsequent degradation following fusion with the cellular lysosomes throughout the lifecycle of an organism in a caspase-independent manner $(8,27)$. Autophagy thus maintains cellular homeostasis throughout the lifecycle of an organism. Recently, many reports have shown that chemotherapeutic agent-induced autophagy was associated with upregulation and processing of LC3/Beclin-1 coupled with induction of Atgs, and its recruitment to the autophagosomes $(28,29)$. In the present study, OB did not alter the expression level of Beclin-1; however, the Atg3 and Atg5 levels, and the autophagic form of LC3 were increased after OB treatment indicating that OB also induced autophagy in A549 cells. 
A
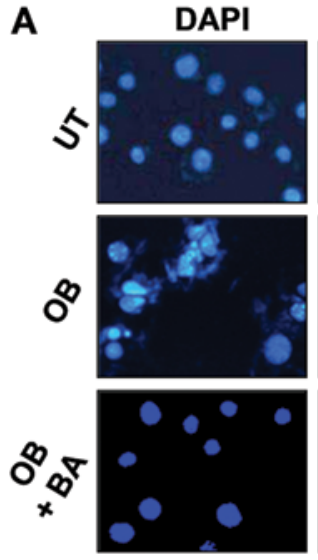

LC3
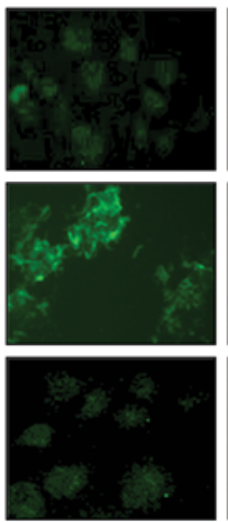
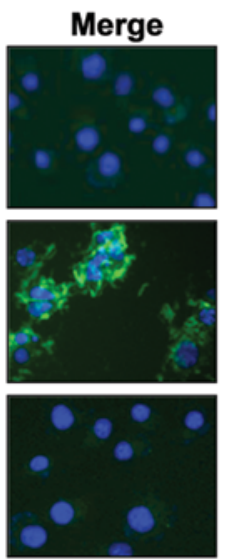

B
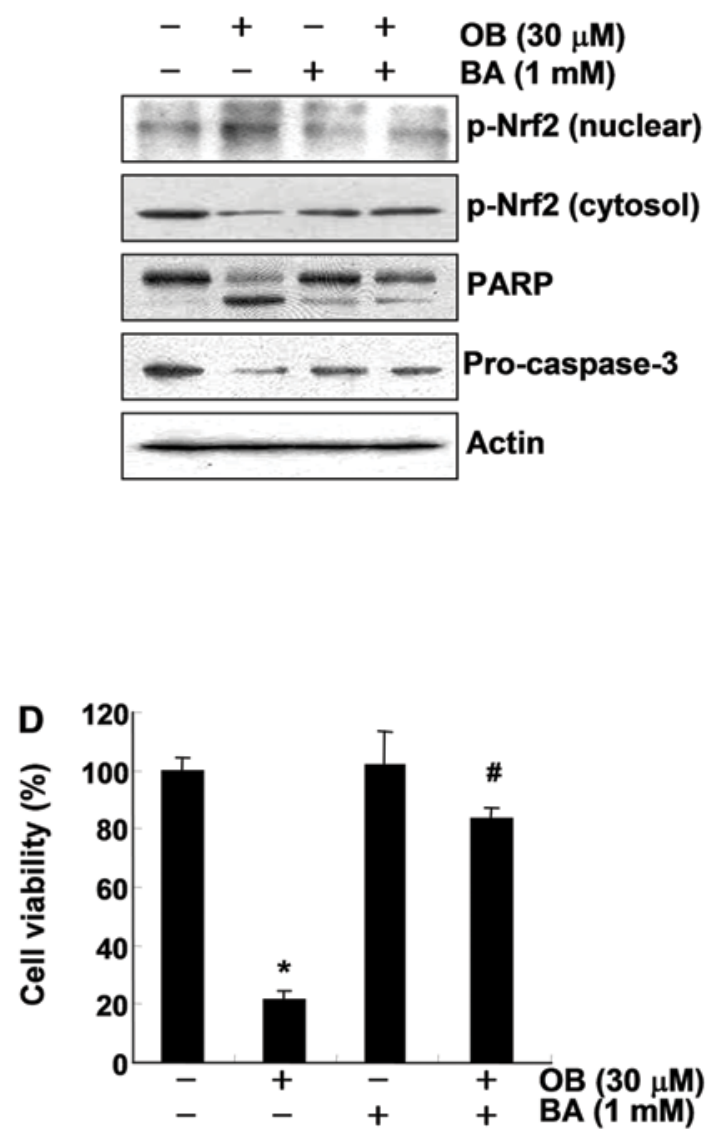

Figure 7. Effects of an autophagy inhibitor, bafilomycin A1, on OB-induced apoptosis in A549 cells. (A) Cells were preincubated with $1 \mu \mathrm{M}$ bafilomycin A1 (BA) for $2 \mathrm{~h}$ and then treated with $30 \mu \mathrm{M}$ OB for $24 \mathrm{~h}$. The cells were fixed and stained with DAPI, anti-LC3 antibody, and then FITC-conjugated anti-rabbit IgG. Images were taken with a laser scanning confocal microscope. (B) The cells were pretreated with $1 \mu \mathrm{M}$ BA for $2 \mathrm{~h}$, and then treated with $30 \mu \mathrm{M} \mathrm{OB}$ for $24 \mathrm{~h}$ and lysed. Equal amounts of proteins were resolved by SDS-polyacrylamide gels, transferred to nitrocellulose and probed with the indicated antibodies. Actin was used as an internal control. (C and D) The cells grown under the same conditions as (B) were collected. Then the percentage of the (C) sub-G1 population and the (D) cell viability were evaluated with a flow cytometer and MTT assay, respectively. Each point represents the mean \pm SD of three independent experiments. The significance was determined with Student's t-test ( ${ }^{*} \mathrm{p}<0.05$ vs. untreated control; ${ }^{*} \mathrm{p}<0.05$ present vs. absent BA).

Nrf2, an essential member of basic leucine zipper transcription factors, by binding to antioxidant response element (ARE) plays a key physiological role in regulating oxidative stress. Previous studies have reported that activation of the Nrf2-ARE/HO-1 pathway by chemotherapeutic agents has been correlated with preventing inflammatory diseases and cancer (30,31). Recently, Ansari et al (32) also reported that activation of the Nrf2-ARE/HO-1 pathway by chemotherapeutic agents caused protection against hydrogen peroxide-induced cell death. Interestingly, our results show that treatment with OB led to a significant reduction in the phosphorylation levels of Nrf2 proteins and expression of HO-1 proteins, but did not affect the total levels of Nrf2 expression. Furthermore, treatment with $\mathrm{OB}$ resulted in the translocation of $\mathrm{p}-\mathrm{Nrf} 2$ from cytosol to the nucleus in A549 cells. Although further studies are needed, these results indicate that OB-induced cell death is involved in the translocation of $\mathrm{p}-\mathrm{Nrf} 2$ into the nucleus in A549 cells.

Apoptosis and autophagy are two distinct forms of programmed cell death; however, apoptosis is always associated with cell death, while autophagy normally contributes either to cell survival or to cell death. In some cases, apoptosis and autophagy are interconnected positively or negatively (11,33-35). Thus, the molecular mechanism controlling the relationship between apoptosis and autophagy during cancer cell death by certain chemotherapeutic agents must be elucidated. Interestingly, in our study, OB-induced autophagy and OB-induced cell death and growth inhibition were significantly attenuated by pretreatment with an autophagy inhibitor bafilomycin A1. Furthermore, pretreatment with bafilomycin A1 had an impact in suppressing OB-induced downregulation of $\mathrm{p}-\mathrm{Nrf} 2$. Taken together, the present results suggest that OB-induced autophagy functioned as a death mechanism and the Nrf2 signaling pathway is involved in this process in A549 cells.

In conclusion, the present results demonstrated that OB-induced cell death occurs through induction of autophagy as cell death mechanisms and caspase-dependent apoptosis. Although the role of the Nrf2-ARE/HO-1 pathway in OB-induced autophagy remains unknown, promoting autophagy could be an effective strategy for enhancing the antitumor activity of OB in A549 NSCLC cells. 


\section{Acknowledgements}

This study was supported by the National Research Foundation of Korea (NRF) grant funded by the Korea government (2012-0000476 and 2012046358).

\section{References}

1. Corcelle EA, Puustinen P and Jäättelä M: Apoptosis and autophagy: Targeting autophagy signalling in cancer cells 'trick or treats'? FEBS J 276: 6084-6096, 2009.

2. Levine B: Cell biology: autophagy and cancer. Nature 446 745-747, 2007.

3. Fuchs Y and Steller H: Programmed cell death in animal development and disease. Cell 147: 742-758, 2011.

4. Hengartner MO: The biochemistry of apoptosis. Nature 407: 770-776, 2000

5. Okada H and Mak TW: Pathways of apoptotic and non-apoptotic death in tumour cells. Nat Rev Cancer 4: 592-603, 2004.

6. Jin Z and El-Deiry WS: Overview of cell death signaling pathways. Cancer Biol Ther 4: 139-163, 2005.

7. Klionsky DJ and Emr SD: Autophagy as a regulated pathway of cellular degradation. Science 290: 1717-1721, 2000.

8. Gozuacik D and Kimchi A: Autophagy and cell death. Curr Top Dev Biol 78: 217-245, 2007

9. Mizushima N, Levine B, Cuervo AM and Klionsky DJ: Autophagy fights disease through cellular self-digestion. Nature 451: 1069-1075, 2008.

10. Cheng Y, Qiu F, Ye YC, Guo ZM, Tashiro S, Onodera S and Ikejima T: Autophagy inhibits reactive oxygen speciesmediated apoptosis via activating p38-nuclear factor-kappa B survival pathways in oridonin-treated murine fibrosarcoma L929 cells. FEBS J 276: 1291-1306, 2009.

11. Eisenberg-Lerner A, Bialik S, Simon HU and Kimchi A: Life and death partners: apoptosis, autophagy and the cross-talk between them. Cell Death Differ 16: 966-975, 2009.

12. Jemal A, Siegel R, Xu J and Ward E: Cancer statistics, 2010. CA Cancer J Clin 60: 277-300, 2010

13. Gridelli C, Maione P, Ferrara ML and Rossi A: Cetuximab and other anti-epidermal growth factor receptor monoclonal antibodies in the treatment of non-small cell lung cancer. Oncologist 14: 601-611, 2009.

14. Kapp FG, Sommer A, Kiefer T, Dölken G and Haendler B: 5-alpha-reductase type I (SRD5A1) is up-regulated in non-small cell lung cancer but does not impact proliferation, cell cycle distribution or apoptosis. Cancer Cell Int 12: 1, 2012.

15. Park BY, Min BS, Oh SR, Kim JH, Kim TJ, Kim DH, Bae KH and Lee HK: Isolation and anticomplement activity of compounds from Dendropanax morbifera. J Ethnopharmacol 90: 403-408, 2004

16. Chung IM, Kim MY, Park WH and Moon HI: Antiatherogenic activity of Dendropanax morbifera essential oil in rats. Pharmazie 64: 547-549, 2009.

17. Moon HI: Antidiabetic effects of dendropanoxide from leaves of Dendropanax morbifera Leveille in normal and streptozotocininduced diabetic rats. Hum Exp Toxicol 30: 870-875, 2011.

18. Chung IM, Kim MY, Park SD, Park WH and Moon HI: In vitro evaluation of the antiplasmodial activity of Dendropanax morbifera against chloroquine-sensitive strains of Plasmodium falciparum. Phytother Res 23: 1634-1637, 2009.
19. Kabeya Y, Mizushima N, Ueno T, Yamamoto A, Kirisako T, Noda T, Kominami E, Ohsumi Y and Yoshimori T: LC3, a mammalian homologue of yeast Apg8p, is localized in autophagosome membranes after processing. EMBO J 19: 5720-5728, 2000 .

20. Riccioni R, Pasquini L, Mariani G, Saulle E, Rossini A, Diverio D, Pelosi E, Vitale A, Chierichini A, Cedrone M, Foà R, Lo Coco F, Peschle $\mathrm{C}$ and Testa U: TRAIL decoy receptors mediate resistance of acute myeloid leukemia cells to TRAIL. Haematologica 90: 612-624, 2005.

21. Lee EW, Seo J, Jeong M, Lee S and Song J: The roles of FADD in extrinsic apoptosis and necroptosis. BMB Rep 45: 496-508, 2012.

22. Deveraux QL and Reed JC: IAP family proteins-suppressors of apoptosis. Genes Dev 13: 239-252, 1999.

23. Kroemer G and Reed JC: Mitochondrial control of cell death. Nat Med 6: 513-519, 2000.

24. Constantinou C, Papas KA and Constantinou AI: Caspaseindependent pathways of programmed cell death: the unraveling of new targets of cancer therapy? Curr Cancer Drug Targets 9: 717-728, 2009

25. Wu XX and Kakehi Y: Enhancement of lexatumumab-induced apoptosis in human solid cancer cells by cisplatin in caspasedependent manner. Clin Cancer Res 15: 2039-2047, 2009.

26. Takeda S, Matsuo K, Yaji K, Okajima-Miyazaki S, Harada M, Miyoshi H, Okamoto Y, Amamoto T, Shindo M, Omiecinski CJ and Aramaki H: (-)-Xanthatin selectively induces GADD45 $\gamma$ and stimulates caspase-independent cell death in human breast cancer MDA-MB-231 cells. Chem Res Toxicol 24: 855-865, 2011.

27. Bröker LE, Kruyt FA and Giaccone G: Cell death independent of caspases: a review. Clin Cancer Res 11: 3155-3162, 2005.

28. Pattingre S, Espert L, Biard-Piechaczyk M and Codogno P: Regulation of macroautophagy by mTOR and Beclin 1 complexes. Biochimie 90: 313-323, 2008.

29. Marquez RT and Xu L: Bcl-2: Beclin 1 complex: multiple, mechanisms regulating autophagy/apoptosis toggle switch. Am J Cancer Res 2: 214-221, 2012.

30. Kang KW, Lee SJ and Kim SG: Molecular mechanism of nrf2 activation by oxidative stress. Antioxid Redox Signal 7: 1664-1673, 2005

31. Khan NM, Sandur SK, Checker R, Sharma D, Poduval TB and Sainis KB: Pro-oxidants ameliorate radiation-induced apoptosis through activation of the calcium-ERK1/2-Nrf2 pathway. Free Radic Biol Med 51: 115-128, 2011.

32. Ansari N, Khodagholi $\mathrm{F}$ and Amini M: 2-Ethoxy-4,5diphenyl-1,3-oxazine-6- one activates the Nrf2/HO-1 axis and protects against oxidative stress-induced neuronal death. Eur J Pharmacol 658: 84-90, 2011.

33. Choi KS: Autophagy and cancer. Exp Mol Med 44: 109-120, 2012.

34. Singh P, Godbole M, Rao G, Annarao S, Mitra K, Roy R, Ingle A, Agarwal G and Tiwari S: Inhibition of autophagy stimulates molecular iodine-induced apoptosis in hormone independent breast tumors. Biochem Biophys Res Commun 415: 181-186, 2011.

35. Lee Y, Hong Y, Lee SR, Chang KT and Hong Y: Autophagy contributes to retardation of cardiac growth in diabetic rats. Lab Anim Res 28: 99-107, 2012. 